\title{
RELATIONSHIP OF MICROMOPHOLOGY TO CHARGE STORAGE AND TRANSFER PROPERTIES IN HETEROGENEOUS FUNCTIONAL MATERIALS
}

\author{
K. Reifsnider ${ }^{1}$, Q. Liu ${ }^{1}$, J.M. Adkins ${ }^{1}$, J. Baker ${ }^{1}$, and F. Rabbi ${ }^{1}$, K. Brinkman ${ }^{2}$
}

1 Center for Heterogeneous Functional Materials (HeteroFoaM Center), Department of Mechanical Engineering, University of South Carolina (corresponding-Reifsnider@cec.sc.edu)

\section{Savannah River National Laboratory, Savannah, Georgia}

\begin{abstract}
The present paper is concerned with heterogeneous materials in which the morphology is specifically designed to achieve functional properties. These materials, or material systems, are found in energy systems such as fuel cells and batteries, but also in aeronautical structures such as the conductive skins of commercial airplanes which have polymer composite primary structures. Many of these heterogeneous materials are dielectric and, under certain conditions, conductive. Charge transport and storage in such materials is typically discussed in terms of equivalent (electrical) circuits, without recourse to first principles physics or mechanics. The present paper discusses the transport and storage properties of several examples of such materials from a computational standpoint, and compares some critical predictions with experimental data. Opportunities and needs for improving our understandings and computational capabilities for this class of problems will also be discussed.
\end{abstract}

Keywords: Heterogeneous functional materials, charge distribution.

\section{INTRODUCTION}

The foundation for our present discussion is heterogeneous functional materials, i.e., heterogeneous material systems which actively interact with and often alter or transform the fields applied to them. Examples are found in many common man-made devices such as the electrodes and membranes that make fuel cells, electolyzers, batteries, and separation membranes work, and in a host of natural materials and organisms such as the lungs of animals, living bone and wood, and the cornea of the eye of mammals and other animals. We will refer to such material systems as HeteroFoaMs.[1] The applied fields may be mechanical, thermal, electrical, or electrochemical, applied singularly or in combination. In the present paper we address the computational concepts commonly used to address the calculation of the properties of such functional materials, and more particularly, to design them for specific functional performance. For expedience, we will limit the scope of our discussion to what we will call the generalized compliance of the material system with the applied fields. For mechanical fields, that compliance may be the classical material stiffness (associated with displacement of material points); for electrical fields the compliance may be discussed as permittivity (associated with the dielectric or conductive displacement of charge), etc. 
For ideally homogenous condensed materials, one might expect these properties to be determined by the intrinsic character of the materials alone. Mechanical stiffness can be related, for example, to the appropriate atomic or molecular potentials (at least for linear elastic response), and permittivity (including conduction for static electric fields) can generally be discussed in terms of the mobility, concentration, and polarity of charge carriers such as ions, electrons, holes, vacancies, etc.[2,3] In such a situation, one might hope to be able to compute these intrinsic properties from first principles. However, for "real" materials with internal defects or heterogeneity, such properties are no longer strictly intrinsic. For example, for mechanical response, the extrinsic details of the size, shape, distribution, and even the local interactions of the resulting localized distortions of global applied fields affect the compliance of a specimen. Entire branches of computational science, such as micro-mechanics and the compliance approaches to linear elastic fracture mechanics, are based on considerations of those extrinsic details.[4,5] These computational methods are a strong foundation for mechanical design of materials and engineering structures.

For electrical fields, the development of appropriate local computational methods is less mature, and many existing approaches are not physically based rendering them useless for the design of the materials themselves. This paper will focus on those issues.

\section{APPROXIMATE METHODS BASED ON PHYSICAL HEURISTICS}

HeteroFoaM materials are typically functional mixtures of two or more constituents and often a third void phase. An example is shown in Fig. 1 below.

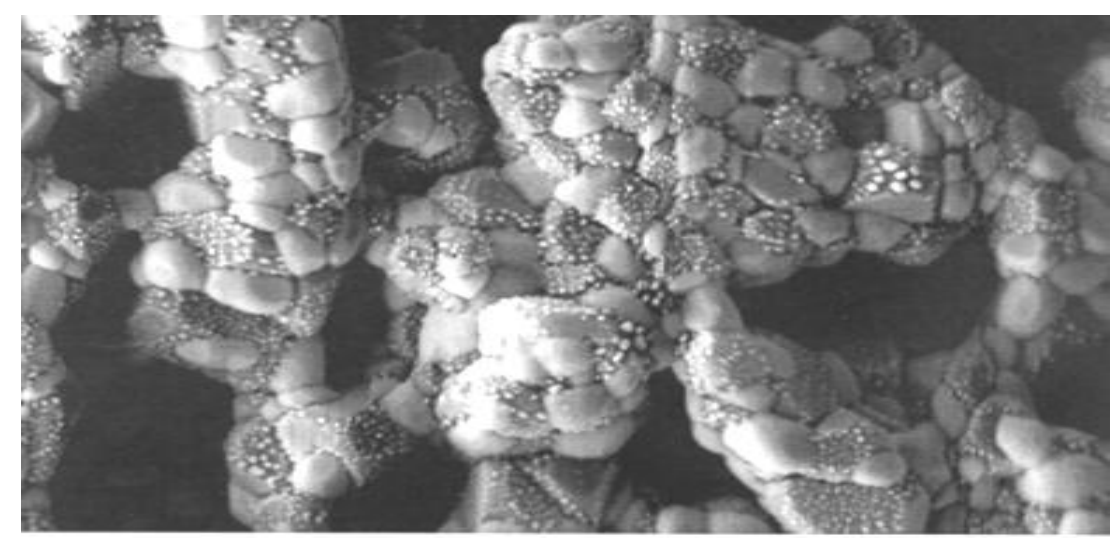

Figure 1. Typical HeteroFoaM microstructure.

In this example, there are ionic conductors (light colored particles), electronic conductors (dark colored particles) and a highly connected void phase that serves to transport fuel in this electrode for an SOFC fuel cell. The local distributions of potentials and the occurrence of charge accumulation at material boundaries greatly influences the conductivity of these current devices and controls their efficiency and power output.[6] If electrochemistry is active on the surfaces of the material, a considerable body of experimental methodology has been developed to assess the magnitude and in some cases the physical mechanisms associated 
with the impedance losses inherent in the functional material system using Electrochemical Impedance Spectroscopy (EIS).[7] If electrochemistry is not involved, a similar methodology associated with just the material response has been widely developed.[8] However, if one asks how to design a better material system, i.e., a better HeteroFoaM, these methods only suggest that one look for constituents with 'better' intrinsic material properties. They suggest nothing about the extrinsic details. Indeed, as we will show, they provide misleading information about the sizes, shapes, and local morphology that are essential to functional performance of heterogeneous systems, and especially as they relate to what the local potentials are and where charge is stored at interfaces.

The essence of this argument is captured by considering the compliance or permittivity of a medium positioned (in a vacuum or other media) between two plates, as shown in Fig. 2 .

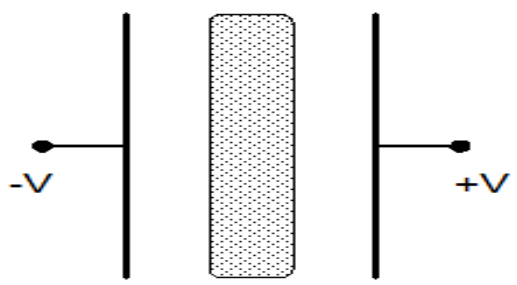

$$
\begin{gathered}
\nabla \cdot \mathrm{D}=0 \\
\mathrm{D}=\varepsilon \cdot \mathrm{E} \\
\mathrm{E}=-\nabla \phi
\end{gathered}
$$

Figure 2. Diagram of conceptual heterogeneous (two phase) material between two plates that provide an electric field (left) and relevant field equations (right)

If the medium between the plates is air or vacuum (with relative permittivity of about unit value), and a dielectric phase is introduced in the interior with a relative permittivity of 5, then the potential distribution in the vacuum (blue line in Fig. 3) will be altered by the polarization of the dielectric phase polarization, as shown by the red line in Fig. 3.

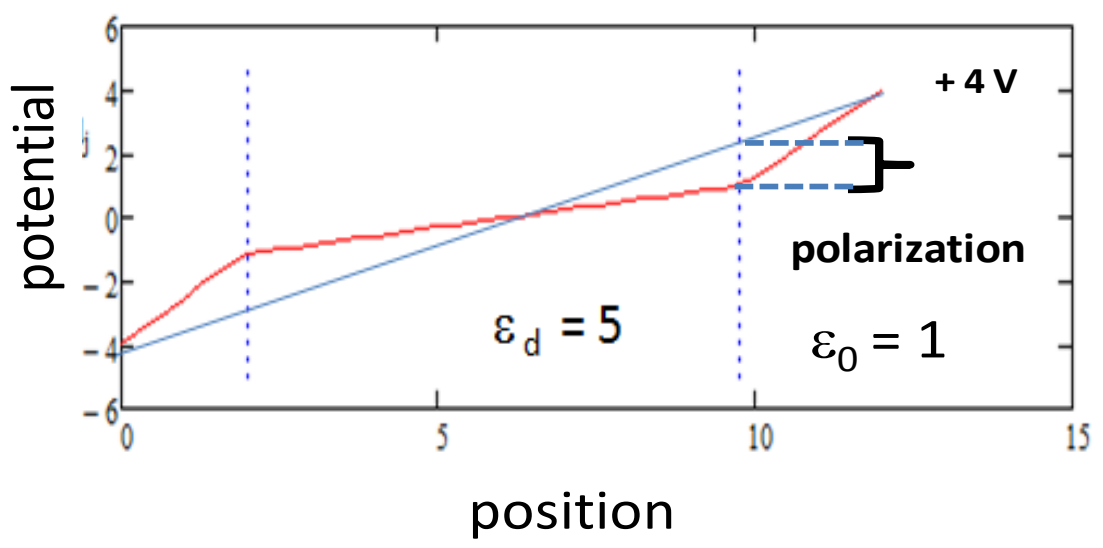

Figure 3. Polarization of a dielectric phase in an insulating medium subjected to a uniform electric field.

Representations of these distributions and the contributions to the dielectric response associated with the charge buildup at the interfaces (typically called Wagner-Sillars polarization) which can be very large and is of special interest to us here are traditionally discussed in terms of equivalent circuits. $[8,9,10]$ These are, by definition, idealized one dimensional 
treatments that group both intrinsic and extrinsic factors into pseudo-electrical-engineering elements such as resistors, capacitors, etc. The resulting response is then discussed in terms of "equivalent circuits." More comprehensive treatments of the conductive and charge displacement behavior of such material systems can be obtained from approximate discrete element solutions of such materials, using for example commercial codes such as COMSOL and others.[11-13] However, most often these representations are based on continuity equations (e.g., Maxwell-Boltzman, etc.) and are not complete solutions for the local fields, rendering them useless for specific discussions of local effects such as surface charge.

In recent work, the authors have developed experimental and analytical methods of recovering those details. Let us consider, for example the problem depicted in Fig. 3 in which the simple (infinite) dielectric plate is replaced by a cylindrical high-dielectric phase in a low dielectric medium, as sketched in Fig. 4 below.

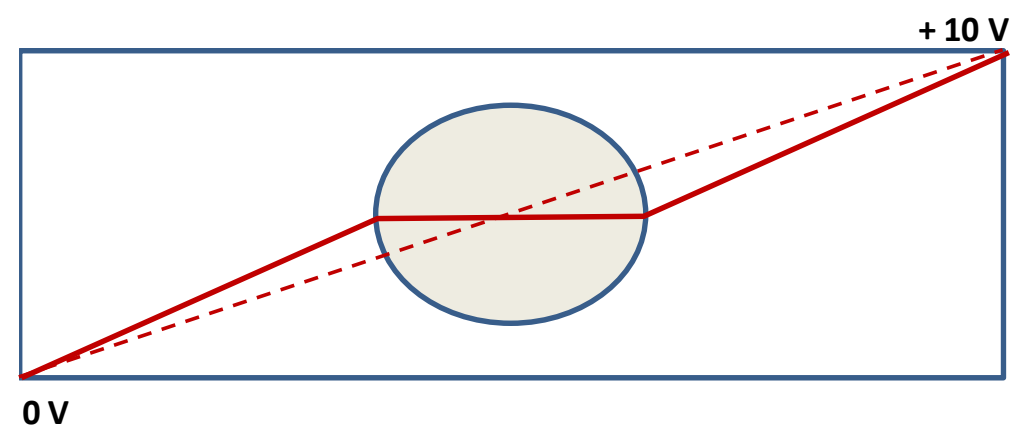

Figure 4. Schematic of a high permittivity rod in a low permittivity medium between two plates.

For this situation we may expect to find the surface charge associated with the polarization, sketched in Fig. 4. Such experiments have been conducted in our laboratories, after adapting an atomic force microscope (AFM) method developed by Qi, et al.[14] for measuring, quantitatively, surface charge and local potential in such samples (to be described in another paper). An example of the experimental setup is sketched below.
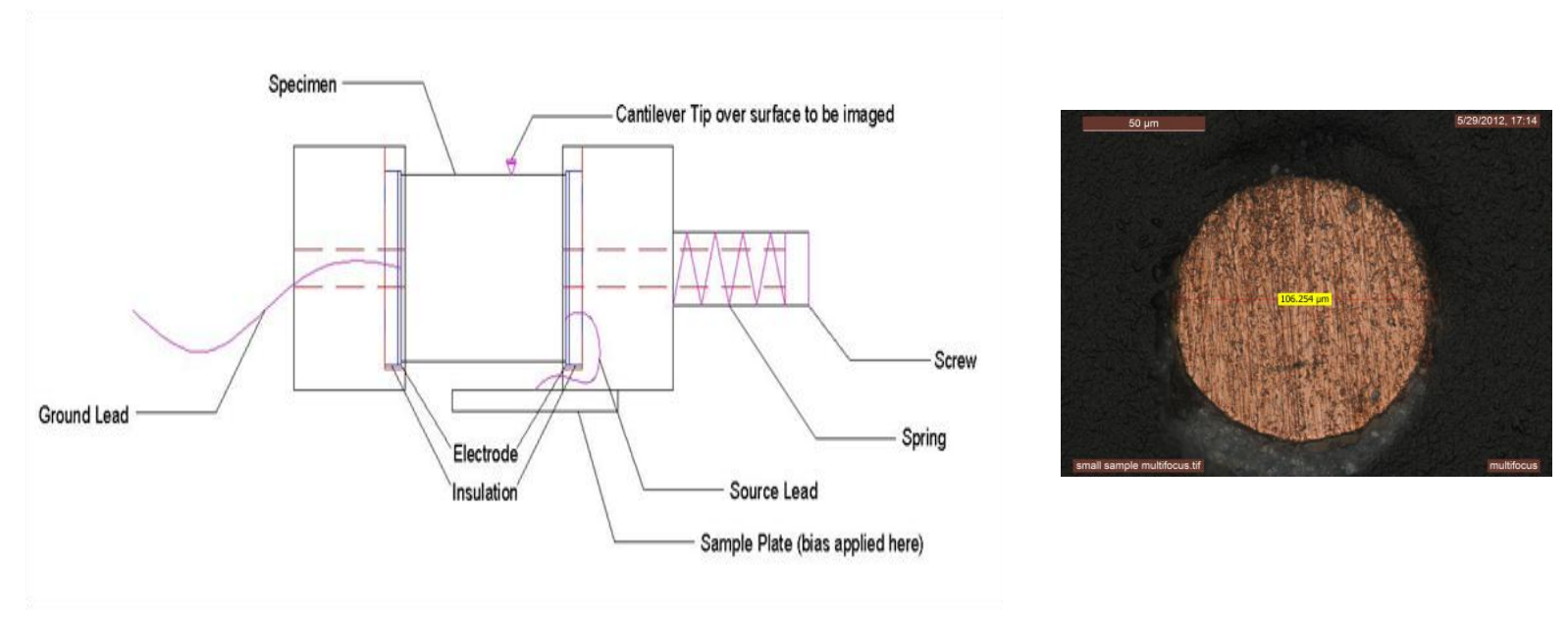

Figure 5. Experimental setup (left) for measuring potential distributions and surface charge in the specimen depicted in Fig. 4; physical test surface (right). 
Using this methodology, surface topology, potentials, and charge distributions were measured over the face of a small rod embedded in epoxy in the configurations shown in Figs. 4 and 5. Figure 6 shows example results for the polarization regions (on the right and left of Figs. 3 and 4).
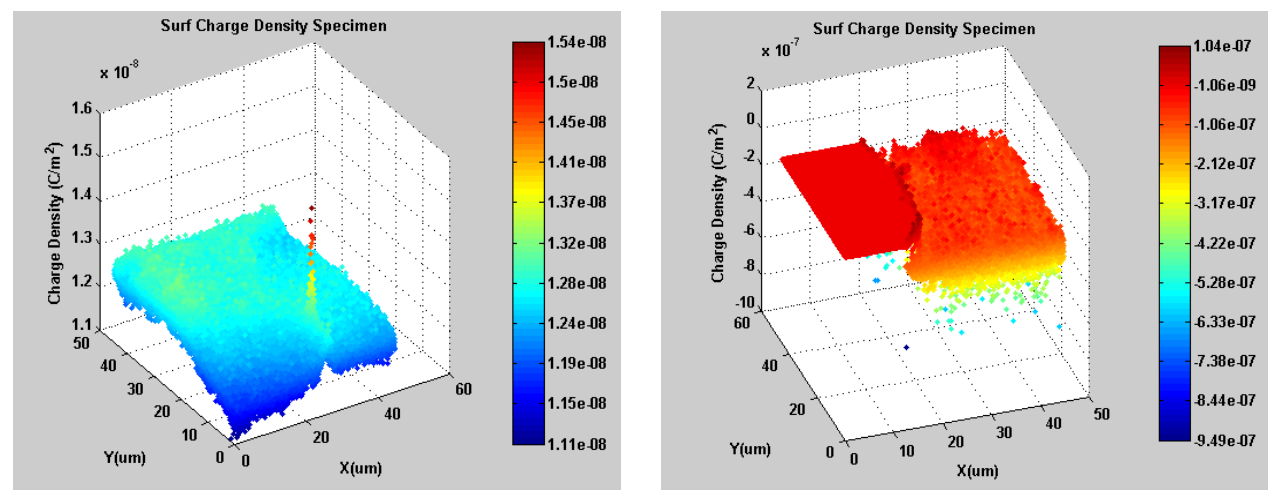

Figure 6. Measured surface charge density in the polarization regions on the left $(+1.2 \mathrm{e}-8 \mathrm{C})$ and right (-9.66e-8C) for an applied specimen bias voltage of $10 \mathrm{~V}$.

These distributions are clearly important to the physical and functional properties of a heterogeneous functional material in which charge transport or storage plays an essential role.
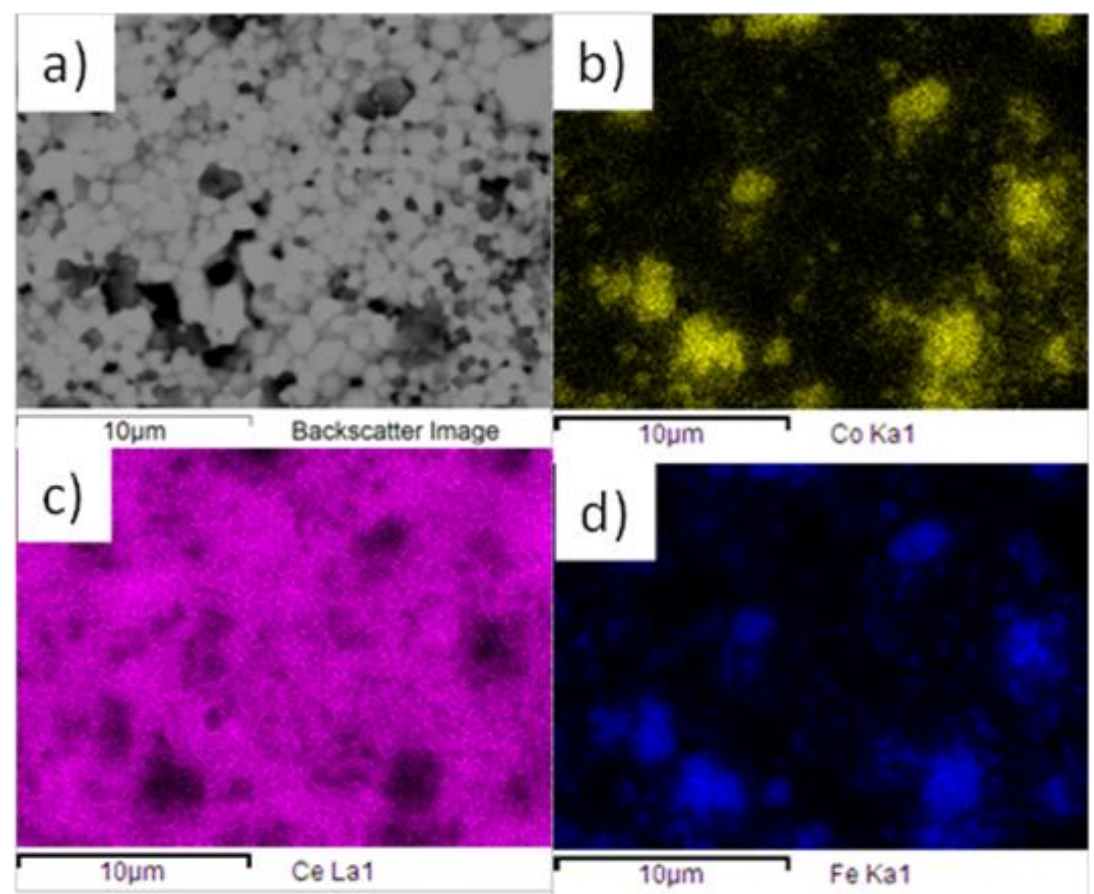

Figure 7. Micrograph of heterogeneous ceramic membrane morphology showing electronic conducting and ionic conducting material phases.

For example, permeation membranes, typically used in combustion devices to support oxyfuel combustion or for partial oxidation reactions involving the production of synthesis gases, require mixed oxygen ion and electronic conduction (MIEC).[15] In addition, solid state separation membranes that transport, for example, oxygen (or other ions) out of molten solid 
streams of oxide fuels or ores are an emerging technology for extraction in the nuclear industry and potentially in the rare materials extraction industry.[16] The morphology of an example dual phase membrane for oxygen separation is shown in Fig. 7 consisting of an electronic conductive phase $\left(\mathrm{CoFe}_{2} \mathrm{O}_{4}\right)$ and an ionic conductive phase $\left(\mathrm{Ce}_{0.9} \mathrm{Gd}_{0.1} \mathrm{O}_{2-\delta}\right) \cdot[17]$

The membrane functional properties (including ionic and electronic conductivity, mass flux transport, etc.) are determined not only by the intrinsic constituent properties, but also by the local extrinsic details such as grain shapes and size, grain boundary properties (including order or disorder), and in some cases the anisotropy of response to a vector applied field. Moreover, it is essential to have a computational approach to the design of such materials for specific response, not just to estimate the global "effective properties" of all possible combinations made by trial and error. The present paper presents such a computational approach.

\section{ANALYSIS METHOD FOR NON-DILUTE HETEROGENEITY}

In the literature, it is widely described that under the condition of matched dielectric time constants between two different phases, no charges accumulate at the interface.[18,19] Another related claim is that the interfacial charges stem from differences in the conductivities of the phases.[8,19] However, this match condition is derived from the simplest model of a one-dimensional two-layer condenser under a direct current (DC) field [18,19] passing through a transient state to a steady state. What is required for more general alternating current (AC) fields, higher dimensions, and curvatures? In this paper, we rigorously analyze the heterogenous dielectric material response in general AC fields to show that those simplified match conditions are invalid for determination of the charge accumulations at the interface.

Consider the physical problem in which a dielectric particle is immersed in a continuous phase in a general AC electric field. The continuous phase (dispersion medium) has an arbitrary dielectric constant $\varepsilon^{\circ}$ and conductivity $\sigma^{\circ}$, where the superscript "o" represents "outside" of the particle, while the dielectric particle has an arbitrary dielectric constant $\varepsilon^{\mathrm{i}}$ and conductivity $\sigma^{i}$, where the superscript " $i$ " represents "inside" the particle. Linear isotropic homogeneity is assumed for both the continuous phase and the particles. For a sinusoidal steady-state electric field, the phasor $\mathrm{V}$ of the potential inside and outside of the particle with specified conductivity, permittivity, and frequency is governed by the complex Laplace's equation

$$
\nabla^{2} \tilde{V}(\mathrm{x}, \mathrm{y}, \mathrm{z})=0
$$

with corresponding interface conditions

$$
\tilde{V}^{o}=\tilde{V}^{i}, \widetilde{\epsilon}^{\mathrm{o}} \frac{\partial \widetilde{V}^{o}}{\partial \mathrm{n}}=\widetilde{\epsilon}^{\mathrm{i}} \frac{\partial \widetilde{V}^{i}}{\partial \mathrm{n}}
$$

where

$$
\tilde{\epsilon}^{\mathrm{i}}=\epsilon+\frac{\sigma}{\mathrm{i} \omega}
$$

For solutions of the potential field having the form 


$$
\mathrm{V}(\mathrm{x}, \mathrm{y}, \mathrm{z}, \mathrm{t})=\operatorname{Re}\{\tilde{V}(\mathrm{x}, \mathrm{y}, \mathrm{z}) \exp (\mathrm{i} \omega \mathrm{t})\}
$$

the total interface charge density can be written as

$$
\sigma_{\mathrm{t}}=-\frac{\partial V^{o}}{\partial \mathrm{n}}+\frac{\partial V^{i}}{\partial \mathrm{n}}
$$

The general spectral analytical solutions outside and inside of a circular dielectric particle can then be written as

$$
\begin{aligned}
& \widetilde{\mathcal{V}}^{o}=\widetilde{b}_{0}+\sum_{n=1}^{\infty}\left[1+\widetilde{K}\left(\frac{r_{p}}{r}\right)^{2 n}\right] r^{n}\left(\widetilde{a}_{n} \cos n \theta+\widetilde{b}_{n} \sin n \theta\right), \\
& \widetilde{\mathcal{V}}^{i}=\widetilde{b}_{0}+\sum_{n=1}^{\infty} \frac{2 \widetilde{\epsilon}^{o}}{\widetilde{\epsilon}^{o}+\widetilde{\epsilon}^{i}} r^{n}\left(\widetilde{a}_{n} \cos n \theta+\widetilde{b}_{n} \sin n \theta\right),
\end{aligned}
$$

where the factor $\widetilde{K}=\frac{\widetilde{\epsilon}-\widetilde{\epsilon}^{i}}{\epsilon^{o}+\widetilde{\epsilon}^{1}}$ and $r_{p}$ is the particle radius, yielding the total interfacial charge density in the form

$$
\sigma_{t}=\sum_{n=1}^{\infty} 2 n r_{p}^{n-1}\left[\operatorname{Re}\left(\widetilde{K} \widetilde{a}_{n} \widetilde{S}\right) \cos n \phi+\operatorname{Re}\left(\widetilde{K} \widetilde{b}_{n} \widetilde{S}\right) \sin n \phi\right] .
$$

The authors have presented a general spectral method for finding solutions to this equation set for various geometries.[20-23] For example, the general analytical solutions outside and inside of an elliptical dielectric particle can be written as

$$
\begin{aligned}
& \tilde{\mathcal{V}}^{o}=\widetilde{b}_{0}+\sum_{n=1}^{\infty} \frac{\left(\widetilde{\epsilon}^{o}+\widetilde{\epsilon}^{i}\right)\left[e^{n\left(\eta+\eta_{p}\right)}+e^{n\left(\eta_{p}-\eta\right)}\right]+\left(\widetilde{\epsilon}^{o}-\widetilde{\epsilon}^{i}\right)\left[e^{n\left(\eta-\eta_{p}\right)}+e^{n\left(3 \eta_{p}-\eta\right)}\right]}{\widetilde{\epsilon}^{o} \cosh n \eta_{p}+\widetilde{\epsilon}^{i} \sinh n \eta_{p}} 2 \widetilde{a}_{n} \cos n \phi \\
& +\sum_{n=1}^{\infty} \frac{\left(\widetilde{\epsilon}^{i}+\widetilde{\epsilon}^{o}\right)\left[e^{n\left(\eta+\eta_{p}\right)}-e^{n\left(\eta_{p}-\eta\right)}\right]+\left(\widetilde{\epsilon}^{i}-\widetilde{\epsilon}^{o}\right)\left[e^{n\left(\eta-\eta_{p}\right)}-e^{n\left(3 \eta_{p}-\eta\right)}\right]}{\widetilde{\epsilon}^{o} \sinh n \eta_{p}+\widetilde{\epsilon}^{i} \cosh n \eta_{p}} 2 \sin n \phi,
\end{aligned}
$$

Then introducing the factors

$$
\widetilde{K_{n}^{a}}=\frac{\widetilde{\epsilon^{o}-\tilde{\epsilon}^{i}}}{\tilde{\epsilon}^{o} \cosh n \eta_{p}+\tilde{\epsilon}^{1} \sinh n \eta_{p}} \text { and } \widetilde{K}_{n}^{b}=\frac{\widetilde{\epsilon^{o}}-\tilde{\epsilon}^{1}}{\tilde{\epsilon}^{o} \sinh n \eta_{p}+\tilde{\epsilon}^{i} \cosh n \eta_{p}}
$$

the total interfacial charge for this case can be written as

$$
h\left(\eta_{p}, \phi\right) \sigma_{t}=\sum_{n=1}^{\infty} 2 n e^{n \eta_{p}}\left[\operatorname{Re}\left(\widetilde{K}_{n}^{a} \widetilde{a}_{n} \widetilde{S}\right) \sinh n \eta_{p} \cos n \phi+\operatorname{Re}\left(\widetilde{K}_{n}^{b} \widetilde{b}_{n} \widetilde{S}\right) \cosh n \eta_{p} \sin n \phi\right.
$$

which reveals that for all particles with different dimensions and curvatures, the interfacial charges are determined by both the real and imaginary parts of $\widetilde{K}$ Since the $\widetilde{K}$ parameters are functions of the material properties, including the permittivity in this case, the interfacial 
charge is determined by all extrinsic aspects of the local geometry and all (real and imaginary) parts of the intrinsic material properties. These equations can be explicitly solved for specific local geometries and material property sets to design the heterogeneous material system to control local surface charge.

Returning to our examples in Figs. 2-6, we can recover the exact charge distributions for our idealized case. Considering the simple estimates of potential that we made in Fig.4, the exact solutions for a cylindrical inclusion are shown in Fig. 8 below. One can see that the idealized estimates are not accurate in either the sense or substance of the correct answer. The potential distributions are not simple linear slopes, for example, and the distribution of potential around the cylinder is not uniform, as shown on the right of Fig. 8. But in the present context, the most remarkable result is shown in Fig. 9, which shows that the interfacial charge on the circumference of the cylinder is quite different as a function of azimuth around the boundary.
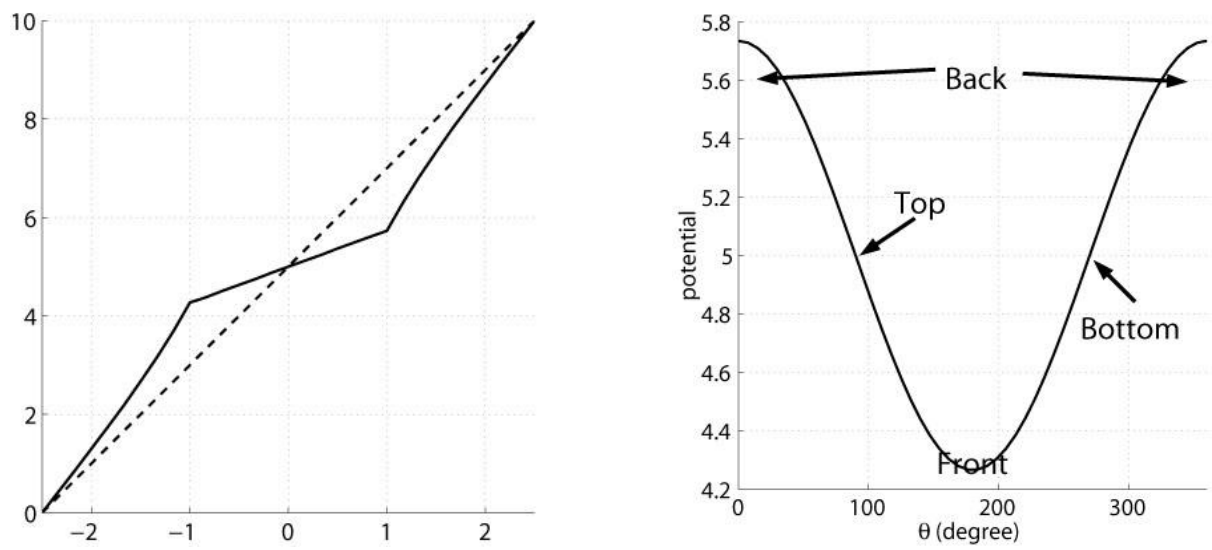

Figure 8. Exact solutions for potential distributions sketched in Fig. 4; potential distribution through the center of the cylinder (left) and around the periphery (right).

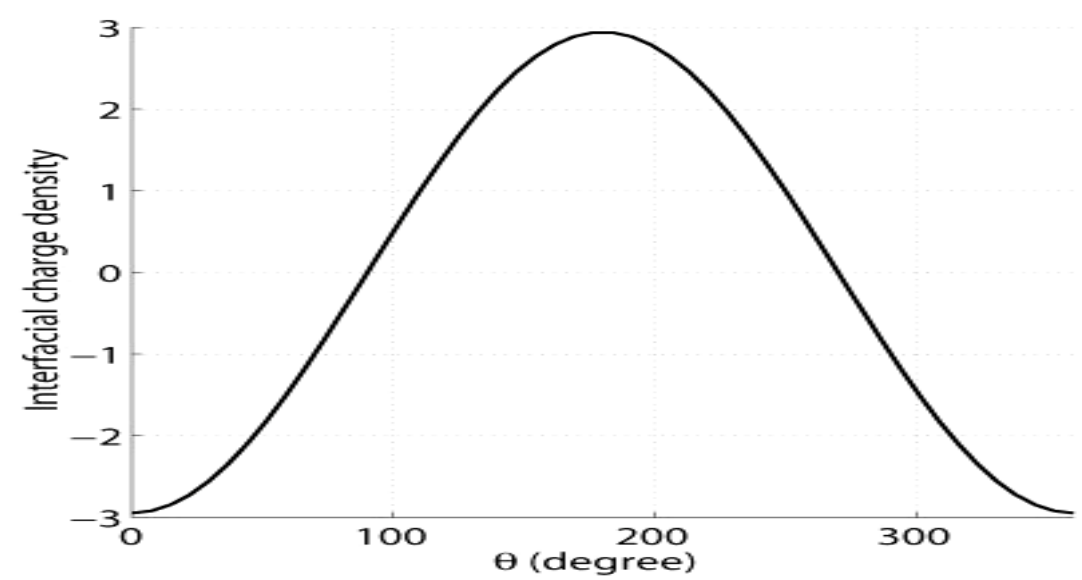

Figure 9. Interfacial charge density distribution around the cylindrical particle shown in Fig. 


\section{CONCLUSIONS}

We report a locally exact analysis for the potential distribution and surface charge distribution in heterogeneous functional materials subjected to electrical fields. This approach makes it possible to design all related aspects of these materials, including the intrinsic material properties and the extrinsic morphology and local structure, to achieve the functional properties such as charge transport and storage which control the success of energy storage and conversion - and many other devices. Further, this method can be applied to non-dilute distributions of phases in a heterogeneous material, an essential and unique characteristic of the approach.[20-23] Finally, we have demonstrated the present concept for cases of general permittivity to electric fields; but a more general formulation for the compliance of heterogeneous materials to other fields (e.g., thermal, mechanical, magnetic, etc.) would be a straight forward extension. This offers many new opportunities for design of HeteroFoaM and other heterogeneous material systems, and presents new challenges for experimental verification and validation.

\section{Acknowledgements}

The authors gratefully acknowledge the support of the Energy Frontier Research Center for Heterogeneous Functional Materials, the HeteroFoaM Center, for support of this research under DoE Grant no. DE-SC0001061 from the Office of Basic Science.

\section{REFERENCES}

1.www.HeteroFoaM.com

2. Dowling, N.E., Mechanical Behavior of Materials, Prentice Hall, New Jersey, 1993

3. Bagotsky, V.S., Fundamentals of Electrochemistry, John Wiley \& Sons, Hoboken, New Jersey, 2006

4. Reifsnider, K.L., Case, S.W., Damage Tolerance and Durability of Material Systems, John Wiley, New York, 2002

5. Irwin G.R., Kies, J.A., Smith, H.L., "Critical energy release rate analysis of fracture strength”. Weld. J. Res. Suppl., 33, 193-198, 1954

6. Huang, K., Goodenough, J., Solid Oxide Fuel Cell Technology, Woodhill, New York, 2009

7. Orazem, M.E., Tribollet, B., Electrochemical Impedance Spectroscopy, John Wiley, New York, 2008

8. Kremer, F., Schonhals, A., Broadband Dielectric Spectroscopy, Springer-Verlag, New York, 2002

9. Wagner, K.W., Arch.Elektrotech., 2, 371, 1914

10. Sillars, R.W., J. Inst. Elect. Eng., 80, 378, 1937

11. Tuncer, E., "Signs of low frequency dispersions in disordered binary dielectric mixtures ". J. Phys. D: Appl. Phys. 37, 334, 2004

12. Rabbi, F., Reifsnider, K., "Relationship of micro-structure morphology to impedance 
in heterogeneous functional materials". Proc. $8^{\text {th }}$ Fuel Cell Science, Engineering and Technology, New York, 33170, 2012

13. Stafford, O.A., Hinderliter, B.R., Croll, S.G., "Electrochemical impedance spectroscopy response of water uptake in organic coatings by finite element methods". Electrochimica Acta 52, 1339-1348, 2006

14. Qi, G., Yang, Y., Yan, H., Guan, L., Li, Y., Qiu, X., et al. "Quantifying Surface Charge Density by Using an Electric Force Microscope with a Referential Structure". Journal of Physics and Chemistry C , 204-207, 2009.

15. Brinkman, K., T. Iijima, and H. Takamura, "The oxygen permeation characteristics of Bi-1 (-) xSrxFeO3 mixed ionic and electronic conducting ceramics". Solid State Ionics. 181(1-2): p. 53-58, 2010.

16. Pal, U.B., D.E. Woolley, and G.B. Kenney, "Emerging SOM Technology for the Green Synthesis of Meltals from Oxides. Journal of Materials". (JOM), 32-35, 2001.

17. Brinkman, K., T. Iijima, and H. Takamura, "The Thickness Dependence of Oxygen Permeability in sol-Gel Derived Ce0.8Gd0.3O2-CoFe2O4 Thin Films on Porous Ceramic Substrates: A Sputtered "Blocking " Layer for Thickness Control". Mater. Res. Soc. Symp. Proc. Volume 1126, Warrendale, PA, 2009, 1126: 145, 2009.

18. Hippel, A.R., Dielectrics and Waves, MIT Press, 1954

19. Prodromakis, T., Papavassiliou, C., "Engineering the maxwell-wagner plorization effect”. Applied Surface Science 255 (2009) 6989-6994

20. Liu, Q., "Physalis method for heterogeneous mixtures of dielectrics and conductors: Accurately simulating one million particles using a PC”. J. Comput. Phys. 230 (2011) 8256-8274.

21. Liu,Q., "Directly resolving particles in an electric field: local charge, force, torque, and applications". Int. J. Numer. Meth. Engng 90 (2012) 537-568.

22. Liu,Q., Reifsnider,K.L., "Heterogeneous mixtures of elliptical particles: Directly resolving local and global properties and responses". Comp. Meth. Appl. Mech. Eng. (2012) Under Review.

23. Liu,Q., Reifsnider,K.L., "Accurate predictions of dielectrophoretic force and torque on many particles with strong mutual field, particle, and wall interactions". Electrophoresis (2012) Under Review. 